\title{
Resonant behavior of a Poisson process driven by a periodic signal
}

\author{
J. Javier Brey, J. Casado-Pascual,* and B. Sánchez \\ Física Teórica, Universidad de Sevilla, Apartado Correos 1065, E-41080 Sevilla, Spain
}

(Received 12 April 1995)

\begin{abstract}
The statistical properties of the residence times of a periodically modulated Poisson process (time-dependent shot noise) on a line segment are analyzed. They show the characteristic features of a resonant behavior, which is similar in many aspects to the stochastic resonance taking place in systems confined in monostable or multistable potentials. The dependence of the mean residence time on both the frequency of the periodic stimulus and its amplitude is studied in detail. The behavior of this parameter also displays the effects associated with the interplay between noise and deterministic modulation.
\end{abstract}

PACS number(s): 05.40. $+\mathrm{j}, 82.20 . \mathrm{Mj}$

\section{INTRODUCTION}

The study of the effects which appear as a consequence of the interaction of noise and periodic stimuli has attracted a considerable interest over the past decade or so. The best known and understood phenomenon is the so-called stochastic resonance, which consists in the enhancement of the response to a small periodic signal by noise in a nonlinear system $[1,2]$. In fact, most of the treatments to date deal with nonlinear systems, where the cooperative effects of the coupling between deterministic and random dynamics were expected to be interesting. Nevertheless, it has been shown that similar and partially unexpected cooperative features also appear in linear systems. In particular, Fletcher, Havlin, and Weiss [3] have described the response of a one-dimensional random walker on a line connecting two traps to a sinusoidally time-dependent stimulus. They show that the mean residence time as a function of the frequency of the field goes through a minimum. More recently, Bulsara, Lowen, and Rees [4] have considered a periodically modulated Wiener process with an absorbing boundary. The behavior they obtain for the response bears a great resemblance to stochastic resonance, although major differences exist in the physical characterization of their model and of bistable systems. It is interesting to notice that the works reported in both papers, Ref. [3] and Ref. [4], were motivated by the modeling of biological processes, namely, pulse field electrophoresis [5] in the former and the response of sensory neurons in the latter [6].

In the present paper we study the response properties to periodic external stimuli of a very simple model. It is a one-dimensional random walk model, like the two mentioned above. In fact, many of the cooperative ef-

\footnotetext{
*Permanent address: Matemáticas Aplicadas II, E.U. Politécnica, Universidad de Sevilla, Virgen de Africa 7, E41011 Sevilla, Spain.
}

fects we will discuss are very similar to the behaviors previously reported $[3,4,7]$, although some significant differences also occur. The theory of first-passage times in stationary one-dimensional random walks is now well established and exact expressions have been worked out for their statistical properties $[8,9]$. Nevertheless, the methods developed do not apply in general when an external time-dependent field is present. For this reason, Fletcher et al. use numerical and perturbative techniques to study the mean first-passage time in their model. On the other hand, Bulsara et al. consider a continuous infinite random walk with only one absorbing boundary, and they are able to construct the exact solution of the FokkerPlanck equation for the distribution function of the variable by means of the method of images.

Let us also point out for completeness that the mean residence time in a line segment presents as well a resonantlike behavior when the state of the lattice fluctuates [10]. In this case, it has a minimum as a function of the correlation time characterizing the fluctuations. This phenomenon has been termed resonant activation [11]. The main difference a priori with the resonant effects mentioned above, which are the ones studied here, is the absence of any external deterministic force in the resonant activation. The latter is due to the interaction of two noise contributions.

The model we will deal with is a sinusoidally modulated Poisson process, in which the walker can move in only one direction. Therefore the residence time in a finite segment can be defined by means of only one absorbing boundary. The simplicity of the model permits us to solve exactly the associated master equation and to study its response properties analytically, without introducing any approximation. This allows a better understanding of the origin of the resonant effects which are observed. In particular, we will analyze in detail the relevant role played by the initial phase of the periodic stimulus, a point that has not been addressed up to now. The model can be useful to describe a wide variety of phenomena in very diverse areas. For instance, it is possible that the voltage of a membrane controlling some neural firing events [12] monotonically increases in time until reaching 
a threshold value at which the firing event occurs. If the successive increases of the voltage are assumed to take place at biased random moments, our model can be used to describe its evolution.

The presentation is organized as follows. In the next section the model is formulated and exact explicit expressions are given for the first-passage time distribution function (FPTDF) and the mean first-passage time (MFPT). The structure of the FPTDF is analyzed in Sec. III, where it is seen to present many of the characteristic features that have been studied in noisy bistable systems in the presence of deterministic modulation. The MFPT is discussed in Sec. IV. It is shown to exhibit a resonantlike behavior when considered as a function of the frequency of the external stimulus. The shape of the function strongly depends on the initial phase of the stimulus. This parameter also plays an important role in determining the behavior of the MFPT when the amplitude of the external perturbation is varied. In some cases, the MFPT makes transitions from a monotonic decrease with increasing amplitude of the sinusoidal term to a monotonic increase with this parameter as the frequency of the signal changes. Finally, in the last section, we summarize some of the main conclusions of this work. Also, a brief reference is made to the power spectral density which is obtained when a simple deterministic mechanism of reinjection for the walker is introduced. Its properties bear again a great resemblance to those found in bistable models.

\section{THE MODEL}

The model we will consider is a continuous-time random walk on a one-dimensional lattice. The walker is restricted to move in a given direction and it is submitted to a time-dependent external field. The probability $p_{n}(t)$ of finding the random walker at site $n$ at time $t$ obeys the master equation

$$
\frac{\partial}{\partial t} p_{n}(t)=\alpha(t)\left[p_{n-1}(t)-p_{n}(t)\right]
$$

where the transition rate $\alpha(t)$ is non-negative for all times. This equation can be easily solved using, for instance, the method of the generating function [8] and the general solution reads

$$
p_{n}(t)=\sum_{n^{\prime}=-\infty}^{n} \frac{p_{n^{\prime}}(0)}{\left(n-n^{\prime}\right) !}[I(t)]^{n-n^{\prime}} e^{-I(t)}
$$

with

$$
I(t)=\int_{0}^{t} d t^{\prime} \alpha\left(t^{\prime}\right)
$$

In what follows we will be concerned with a walker which is initially in the line segment $(0, N)$. Therefore for $n \geq$ $N$ the sum in Eq. (2) extends from $n^{\prime}=0$ to $n^{\prime}=N$. Our main interest will be in the properties of the arrival times to site $N+1$. Because of the Poisson nature of the random process we are considering, the walker never returns to a site after leaving it. Thus the first-passage time distribution function $f(t)$ is simply

$$
f(t)=\alpha(t) p_{N}(t),
$$

and the mean first passage time is

$$
\tau=\int_{0}^{\infty} d t t f(t)=\sum_{n=0}^{N} \int_{0}^{\infty} d t p_{n}(t) .
$$

Use of Eq. (2) yields

$$
\tau=\sum_{n=0}^{N} \frac{p_{n}(0)}{\Gamma(N+1-n)} \int_{0}^{\infty} d t \Gamma(N+1-n, I(t)),
$$

where $\Gamma(n, x)$ is the incomplete gamma function [13]. Now we particularize for a sinusoidally oscillating external field and take

$$
\alpha(t)=p+\epsilon \sin (\omega t+\phi)
$$

The parameter $\epsilon$ measures the amplitude of the periodic stimulus and it is restricted to the interval $0 \leq \epsilon \leq p$. Besides, we also consider the specific initial condition

$$
p_{n}(0)=\delta_{n, 0}
$$

Then, the FPTDF reads

$$
f(t)=\frac{\alpha(t)}{N !}[I(t)]^{N} e^{-I(t)}
$$

with

$$
I(t)=p t-\frac{\epsilon}{\omega}[\cos (\omega t+\phi)-\cos \phi] .
$$

For given values of the parameters, $I(t)$ is an increasing function of $t$. Although Eq. (9) provides an exact explicit expression for $f(t)$, and will be used in most of the calculations, it is convenient for future use to consider also the linear stimulus approximation. Expansion of Eq. (9) to first order in $\epsilon$ gives

$$
\begin{aligned}
f^{(l)}(t)= & f_{0}(t)\left[1+\frac{\epsilon}{p}(\sin (\omega t+\phi)\right. \\
& \left.+\frac{p t-N}{\omega t}([\cos (\omega t+\phi)-\cos \phi])\right],
\end{aligned}
$$

where

$$
f_{0}(t)=\frac{p}{N !}(p t)^{N} e^{-p t}
$$

is the FPTDF for the Poisson process in absence of the periodic stimulus. The corresponding linear approximation for the MFPT can be derived by linearizing Eq. (6) or by introducing Eq. (11) into Eq. (5). The result is

$$
\tau^{(l)}=\tau_{0}-\frac{\epsilon}{\omega p}\left[\cos \phi-\frac{\cos \left((N+1) \arctan \frac{w}{p}+\phi\right)}{\left(1+\frac{\omega^{2}}{p^{2}}\right)^{\frac{N+1}{2}}}\right] .
$$


Here $\tau_{0}$ is the MFPT for the distribution $f_{0}(t)$, i.e., $\tau_{0}=$ $(N+1) p^{-1}$.

\section{FIRST-PASSAGE TIME DISTRIBUTION FUNCTION}

The temporal behavior of the FPTDF depends on the value of the frequency of the perturbation. We have carried out an asymptotic analysis of Eq. (9) in the limit of large $N$. The results remain qualitatively valid for $N$ not too small. In Appendix A we give some details of the calculations and here we merely present the main conclusions. At large frequencies, $\omega N^{1 / 2} p^{-1}$ of the order of unity or larger, the FPTDF is given by

$$
f(t)=\frac{N^{-1 / 2}}{\sqrt{2 \pi}} \alpha(t) \exp \left[-\frac{1}{2 N}[I(t)-N]^{2}\right] .
$$

The dominant region of the distribution is determined by the condition $I(t) \sim O(N)$, which leads to $t \sim O\left(\tau_{0}\right)$. In this region, the FPTDF presents a series of peaks due to the $\alpha(t)$ factor. For lower frequencies, $\omega N^{1 / 2} p^{-1} \ll 1$, Eq. (14) reduces to the Gaussian form

$$
f(t)=\frac{1}{\sqrt{2 \pi} \sigma} \exp \left[-\frac{\left(t-t_{m}\right)^{2}}{2 \sigma^{2}}\right],
$$

where

$$
\sigma=\frac{N^{1 / 2}}{p+\epsilon \sin \left(\omega t_{m}+\phi\right)}
$$

and $t_{m}$ is the time at which the function

$$
h(t)=\exp \left[-\frac{1}{2 N}[I(t)-N]^{2}\right]
$$

is maximum, i.e., it is the solution of the equation $I\left(t_{m}\right)=N$ or

$$
p \omega t_{m}-\epsilon\left[\cos \left(\omega t_{m}+\phi\right)-\cos \phi\right]=\omega N,
$$

which, in general, has to be solved by means of graphical or numerical methods. Therefore $f(t)$ consists now of a single peak located at $t=t_{m}$. Finally, in the range of very low frequencies, defined by $\omega N p^{-1} \ll 1$, the solution of Eq. (18) is

$$
t_{m}=\frac{N}{p+\epsilon \sin \phi}
$$

and Eq. (16) simplifies to

$$
\sigma=\frac{N^{1 / 2}}{p+\epsilon \sin \phi}=\frac{t_{m}}{N^{1 / 2}} .
$$

We notice that in this very low frequency limit the FPTDF does not depend on the frequency of the stimulus.

When $N$ is not asymptotically large, the separation of the frequency scales is not well defined, but the three ranges discussed above are clearly identified as illustrated by Figs. 1 and 2. In Fig. 1 the FPTDF computed from Eq. (9) is shown for $N=50, p=1, \epsilon=0.3, \phi=\pi / 2$, and $\omega=2$. Also plotted are Eq. (14) and $f_{0}(t)$. The function $f(t)$ oscillates around $f_{0}(t)$, in agreement with the above discussion. In Fig. 2 the values of the parameters are the same with the only difference that now it is $\omega=0.01$. The curves presented correspond to Eqs. (9), (15), and (12), respectively. The differences between the exact and the asymptotic expression disappear on the scale of the figures if one takes $N=100$.

The multipeaked structure of the FPTDF follows directly from the presence of noise and has been extensively studied in the case of modulated noisy bistable systems [2]. Very recently, Bulsara, Lowen, and Rees [4] found the same structure in a periodically driven continuous random walk with an absorbing boundary. In fact, their model is similar to ours in the sense that the presence of a positive drift guarantees that the walker will reach the boundary for all values of the parameters, contrary to what happens, for instance, in bistable models of stochastic resonance [2].

The exact location of the peaks of the FPTDF is a quite complicated function of all the system parameters. Nevertheless, the position, $t_{l}$, of those peaks located in the vicinity of $t_{m}$ is given by

$$
t_{l}=\frac{1}{\omega}\left(2 \pi l+\frac{\pi}{2}-\phi\right),
$$

where $l$ is a positive integer. More precisely, Eq. (21) holds in the limit

$$
\frac{p^{3}}{\omega \epsilon N}\left|t_{l}-t_{m}\right| \ll 1
$$

A proof of this is sketched in Appendix B. In practice, Eq. (21) provides a good approximation for all the peaks which show up in the relevant part of the FPTDF, as is

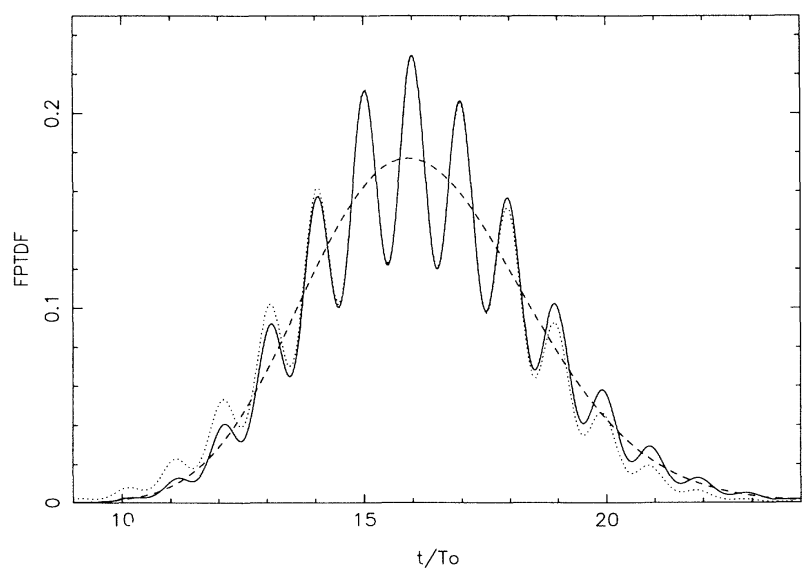

FIG. 1. FPTDF versus normalized time $t / T_{0}$, where $T_{0}=2 \pi / \omega$. For all curves it is $N=50, p=1, \omega=2$, and $\phi=\pi / 2$. For the dashed curve it is $\epsilon=0$, Eq. (12), and for the other two $\epsilon=0.3$. The solid curve has been plotted using the exact expression, Eq. (9), and the point curve using the asymptotic expression, Eq. (14). 


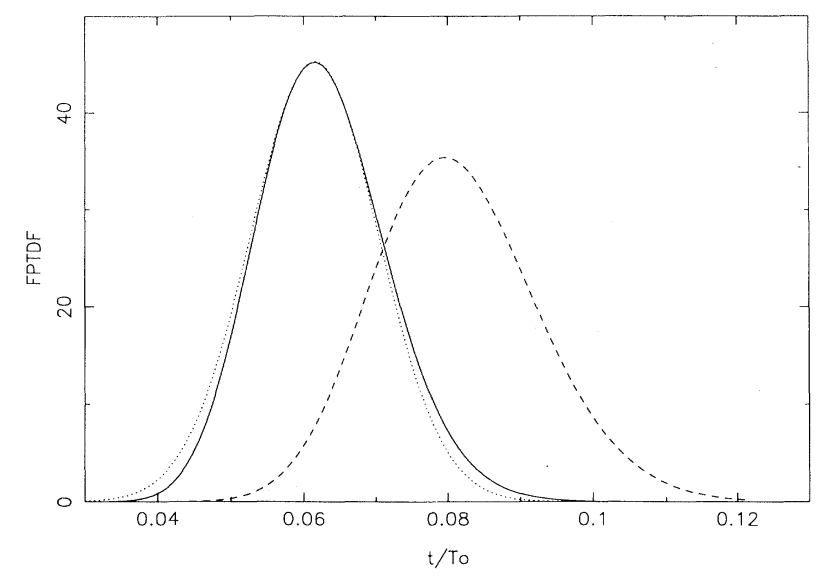

FIG. 2. FPTDF versus time for the same values of the parameters as in Fig. 1, with the only differences that now it is $\omega=0.01$ for all curves and the point curve corresponds to Eq. (15).

the case, for instance, in Fig. 1. Of course, when $t_{m}=$ $\frac{1}{\omega}\left(2 \pi k+\frac{\pi}{2}-\phi\right)$, with $k$ a positive integer, there is a peak at $t_{m}$ and it is the highest one of the distribution.

If the FPTDF is considered as a function of the constant part $p$ of the transition rate for an arbitrary given time $t$, it is found by direct differentiation of Eq. (9) that

$$
\left(\ln f_{p}\right)^{\prime} \equiv \frac{\partial \ln f}{\partial p}=\frac{1}{\alpha}+\frac{N t}{I}-t
$$

and

$$
\left(\ln f_{p}\right)^{\prime \prime} \equiv \frac{\partial^{2} \ln f}{\partial p^{2}}=-\frac{1}{\alpha^{2}}-\frac{N t^{2}}{I^{2}}
$$

These equations indicate that $\left(\ln f_{p}\right)^{\prime}$ has two vertical asymptotes at $p=p_{1}$ and $p=p_{2}$, defined by $\alpha\left(p_{1}, t\right)=0$ and $I\left(p_{2}, t\right)=0$, respectively. Except at these singularities, $\left(\ln f_{p}\right)^{\prime}$ is a monotonic decreasing function of $p$. Furthermore, $\left(\ln f_{p}\right)^{\prime}$ tends to $-t$ as $p$ goes to infinity. As a consequence, $\left(\ln f_{p}\right)^{\prime}$ has a zero for some critical value $p=p_{c}$ inside the interval $\max \left(p_{1}, p_{2}\right)<p_{c}<\infty$. For this value, $f(t)$ presents a maximum as $p$ is increased. We have shown above that the location of the peaks inside the relevant region is approximately independent of $p$. It follows that the heights of the peaks will go through a maximum as functions of $p$. The critical value $p_{c, l}$ at which the peak located at $t_{l}$ presents its maximum will verify the equation

$$
I\left(p_{c, l}, t_{l}\right)+t_{l} \alpha\left(p_{c, l}, t_{l}\right)\left[N-I\left(p_{c, l}, t_{l}\right)\right]=0 .
$$

This is a second degree equation for $p_{c, l}$. One of the solutions is always smaller than $\epsilon$ and, therefore, must be eliminated. The other one is

$$
p_{c, l}=\frac{1}{2 t_{l}}\left[A+\left(B^{2}+4 N\right)^{1 / 2}\right]
$$

where

$$
A=N+1-\frac{\epsilon \cos \phi}{\omega}-\epsilon t_{l}
$$

and

$$
B=N-1-\frac{\epsilon \cos \phi}{\omega}+\epsilon t_{l} .
$$

It happens that the right hand side of Eq. (26) is smaller than $\epsilon$ for some values of the parameters, indicating that in those cases the maximum cannot be observed in the parameter region in which the model is defined. From the analysis of Eqs. (25) and (26) it can be easily proved that

$$
\frac{\partial p_{c, l}}{\partial t_{l}}<0
$$

and this allows the determination of the boundary value $\hat{t}_{M}$ of $t_{l}$ for which the maximum of the peak will be reached upon varying $p$ in the interval $\epsilon<p<\infty$. Вy making $p_{c, l}=\epsilon$ in Eq. (26) one gets

$$
\hat{t}_{M}=\frac{C+\left(C^{2}+8 D\right)^{1 / 2}}{4 \epsilon \omega}
$$

where

$$
C=(2 N+1) \omega-2 \epsilon \cos \phi
$$

and

$$
D=\epsilon \omega \cos \phi
$$

So, the maximum can be reached for those peaks such that $t_{l} \leq t_{M}$. In Fig. 3 we plot the height of the peaks corresponding to $l=3,4$, and 5 , respectively, for a particular set of system parameters given in the figure caption. For them it is $\hat{t}_{M}=102.5$, which leads to $M=32.62$, i.e., the above discussion predicts that the last peak going

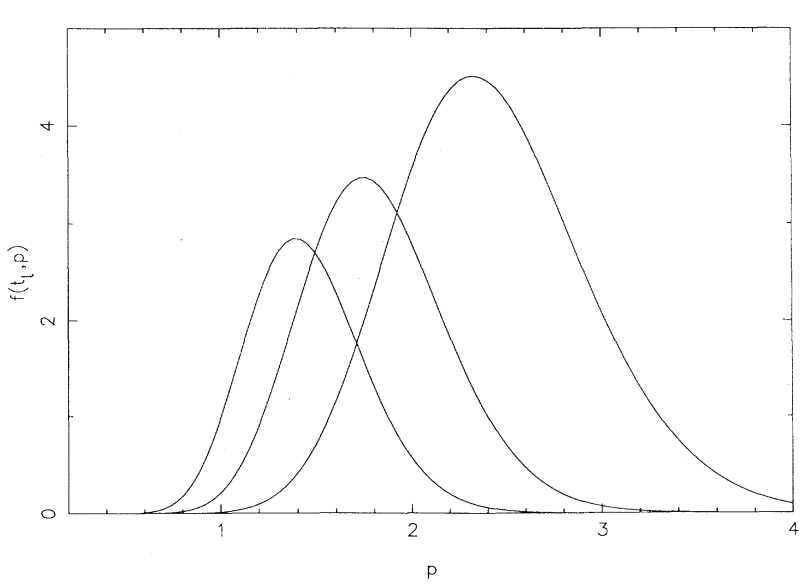

FIG. 3. FPTDF peak heights versus $p$ for $N=20, \omega=2$, $\epsilon=0.2$, and $\phi=\pi / 2$. From left to right the curves correspond to peak numbers $l=5, l=4$, and $l=3$. 
through a maximum is the one located at $t=t_{32}$. This is very close to the exact value obtained numerically, which is $t=t_{33}$, as seen from the results presented in Fig. 4. Notice that when a peak reaches its maximum height, it does not mean that it is the highest one of the FPTDF for that value of $p$.

The above resonant effect in the peak heights of the FPTDF is well known in noisy bistable dynamical systems, as well as in Wiener processes with drift and an absorbing boundary [4]. Moreover, in the latter, an antiresonant behavior of the FPTDF was pointed out by Bulsara, Lowen, and Rees in Ref. [4]. The height of the maximum of the distribution presents a minimum as a function of noise. A similar effect does not take place in our model. For $\epsilon=0$ this can be seen by differentiation of Eq. (12). The height of the distribution is a monotonic increasing function of $p$. The same happens for $\epsilon>0$, as can be shown in the following way. For a given value of $p$, we can set the frequency such that a particular peak $l$ is the highest of the distribution. This amounts to taking the value of $\omega=\hat{\omega}_{l}$ such that the enveloping of the peaks of $f(t)$ has its maximum at $t=t_{l}$. The equation of the enveloping, $\hat{f}(t)$, is obtained by making $\cos (\omega t+\phi)=0$ and $\sin (\omega t+\phi)=1$ in the expression of $f(t)$. Using Eq. (9) one gets

$$
\frac{\partial \ln \hat{f}(t)}{\partial t}=p \frac{N-p t-\frac{\epsilon}{\omega} \cos \phi}{p t+\frac{\epsilon}{\omega} \cos \phi} .
$$

Therefore $\hat{f}(t)$ will have a maximum at $t=t_{l}$ if the frequency is

$$
\hat{\omega}_{l}=\frac{p}{N}\left(2 \pi l+\frac{\pi}{2}-\phi+\frac{\epsilon}{p} \cos \phi\right) .
$$

Changing $p$ leads to FPTDF's which all present their maximum at the $l$ peak, and the height of this maximum, $f_{\max }(p)$, is obtained by substituting the values of $t_{l}$ and $\hat{\omega}_{l}$ in Eq. (9). The result is

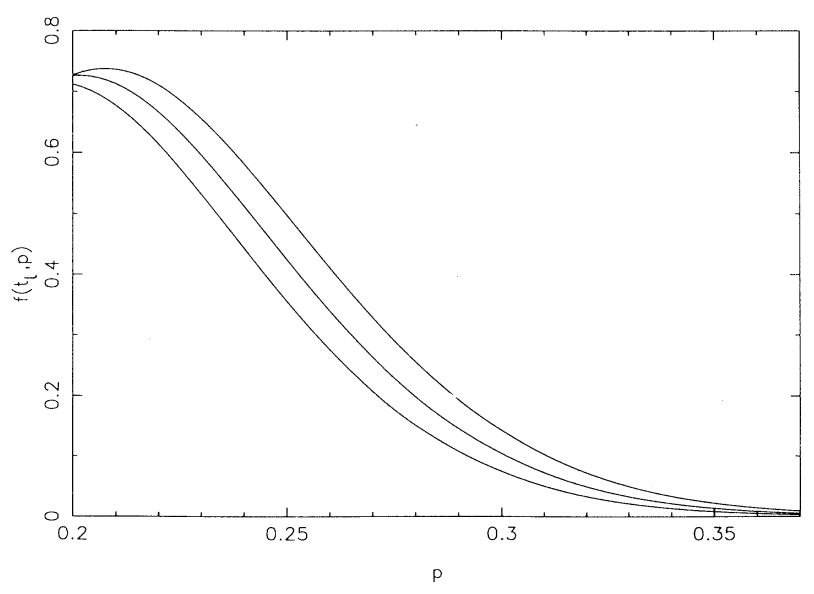

FIG. 4. FPTDF peak heights versus $p$ for the same values of the parameters as in Fig. 3. From left to right the curves correspond to peak numbers $l=35, l=34$, and $l=33$.

$$
f_{\max }(p)=\frac{p+\epsilon}{N !} N^{N} e^{-N}
$$

which is independent of the particular peak $l$ chosen. Since the right hand side of Eq. (35) is a monotonic increasing function of $p$, we get neither a resonant behavior nor an antiresonant one. From this point of view its behavior is different from both bistable systems and the Wiener process discussed in Ref. [4].

\section{RESONANT BEHAVIOR OF THE MEAN FIRST-PASSAGE TIME}

In this section we are going to study the behavior of the MFPT $\tau$ as a function of the several parameters defining the model. We will consider first the dependence on the frequency $\omega$. From Eqs. (6) and (8) one gets

$$
\tau(0) \equiv \tau(\omega=0)=\frac{\tau_{0}}{1+\frac{\epsilon}{p} \sin \phi}
$$

and

$$
\tau(\infty) \equiv \lim _{\omega \rightarrow \infty} \tau(\omega)=\tau_{0},
$$

as expected. It is also obtained that

$$
\left(\frac{\partial \tau}{\partial \omega}\right)_{\omega=0}=-\frac{(N+1)(N+2) \epsilon \cos \phi}{2(p+\epsilon \sin \phi)^{3}} .
$$

It is clear that if the initial slope of $\tau(\omega)$ is negative and $\tau(0) \leq \tau(\infty)$, there must be at least a minimum at some frequency $\omega^{*}$, which, in general, will depend on $N, \epsilon, p$, and $\phi$. On the other hand, if the initial slope is positive, it is also possible for $\tau(\omega)$ to present a minimum, but there must be a maximum before it, i.e., at lower frequencies. For the case of negative initial slope and $\tau(0)>\tau(\infty)$ no conclusion emerges from Eqs. (36)-(38).

Equation (38) shows that the sign of the initial slope of $\tau(\omega)$ is determined by the value of the initial phase $\phi$ of the sinusoidal perturbation. The same parameter also determines whether $\tau(0)$ is larger or smaller than $\tau(\infty)$. For $0 \leq \phi<\pi / 2$ the MFPT always presents a minimum. As mentioned in the Introduction, a similar effect was noticed by Fletcher, Havlin, and Weiss [3] for a random walker that diffuses on a line connecting two traps, and they referred to $\omega^{*}$ as a resonant frequency. Their numerical study was restricted to the value $\phi=0$ and they did not discuss the relevance of the initial phase of the perturbation. Of course, the structure of Eq. (6) shows that $\tau$ as a function of $\omega$ can present not only a first minimum but also a series of peaks. Their origin can be further easier understood if we consider the linear in $\epsilon$ approximation of the MFPT given by Eq. (13). The secondary peaks will be appreciable if the cosine term has time to oscillate before it becomes very small due to the $\left(1+\omega^{2} / p^{2}\right)^{-N / 2}$ factor, i.e., if $N$ is large enough for fixed values of all the other parameters. This is illustrated in Fig. 5, where we have plotted $\tau /(N+1)$ for $N=10$ and $N=50$. In each of the cases two values of the amplitude, $\epsilon=0.1$ and $\epsilon=0.3$, have been considered. In all curves 


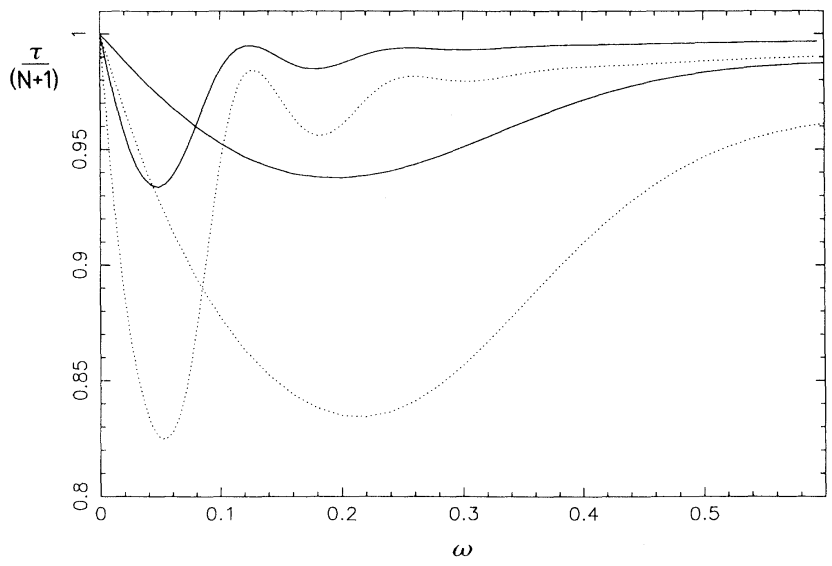

FIG. 5. MFPT divided by $N+1$ versus frequency of the stimulus for $\epsilon=0.1$ (solid curves) and $\epsilon=0.3$ (point curves). For the two upper curves, showing two minima, it is $N=50$, while for the two curves presenting only one minimum it is $N=10$. For all curves $p=1$, and $\phi=0$.

it is $\phi=0$ and $p=1$.

For $\phi=\pi / 2$, the MFPT has an absolute minimum at $\omega=0$, as shown in Fig. 6 for three choices of $\epsilon$. The existence of this minimum has a trivial explanation. For $\phi=\pi / 2$ the transition rate $\alpha(t)$ takes its greatest possible value at $t=0$ and, therefore, the minimum MFPT corresponds to the limit in which $\alpha$ remains constant in time.

We have also investigated the asymptotic interval length dependence of the frequency $\omega^{*}$ at which the first maximum (or minimum) of the MFPT appears. From the asymptotic expressions for $f(t)$ obtained in Appendix A and discussed in Sec. III it follows that the first stationary points of $f(t)$ appear for

$$
\omega^{*} \sim a N^{-1}
$$

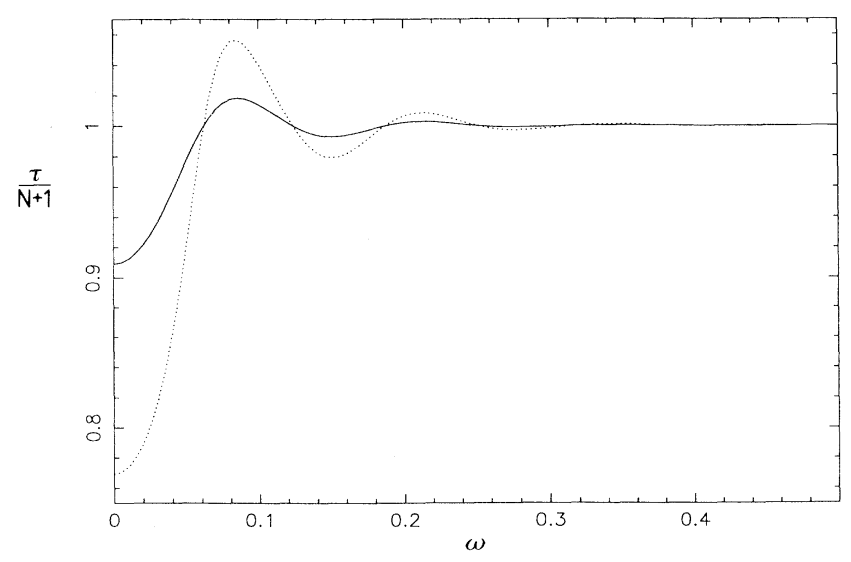

FIG. 6. MFPT divided by $N+1$ versus frequency of the stimulus for $\epsilon=0.1$ (solid curve) and $\epsilon=0.3$ (point curve). For the two curves it is $N=50, p=1$, and $\phi=\frac{\pi}{2}$. where $a$ is of the order of unity. Use of Eqs. (5), (15), and (18) yields

$$
\cos \left(\omega^{*} \tau^{*}+\phi\right)-\cos \phi+\omega^{*} \tau^{*} \sin \left(\omega^{*} \tau^{*}+\phi\right)=0
$$

and

$$
p \tau^{*}+\epsilon \tau^{*} \sin \left(\omega^{*} \tau^{*}+\phi\right)=N,
$$

where $\tau^{*}=\tau\left(\omega^{*}\right)$. The balance of Eq. (40) shows that

$$
\tau^{*} \sim a^{\prime} N
$$

The above asymptotic dependencies on $N$ of $\omega^{*}$ and $\tau^{*}$ seem to be quite general for the resonant behavior of the MFPT for random walks on a line segment $[3,10]$. Substitution of Eqs. (39) and (42) into Eqs. (40) and (41) leads to

$$
\cos \left(a a^{\prime}+\phi\right)-\cos \phi+a a^{\prime} \sin \left(a a^{\prime}+\phi\right)=0
$$

and

$$
p a^{\prime}+\epsilon a^{\prime} \sin \left(a a^{\prime}+\phi\right)=1 .
$$

From this couple of equations one can determine $a$ and $a^{\prime}$ from the values of $p, \epsilon$, and $\phi$. Figures 7 and 8 plot $\omega^{*}$ and $\tau^{*}$, respectively, as functions of $N$ for several values of $\epsilon$. The curves have been obtained by numerical integration of Eq. (6) and for all of them it is $\phi=0$ and $p=1$. The figures clearly confirm the asymptotic behaviors of Eqs. (39) and (42). Moreover, if the slopes for large $N$ are compared with the solutions of Eqs. (43) and (44), an excellent agreement is obtained. The discrepancies are within the numerical errors. Of course, upon solving Eqs. (43) and (44) one has to choose, for each value of $\epsilon$, the solution leading to the smallest positive values of $a$ and $a^{\prime}$. It is also worth mentioning that the equations show that the resonant frequency $\omega^{*}$ is a

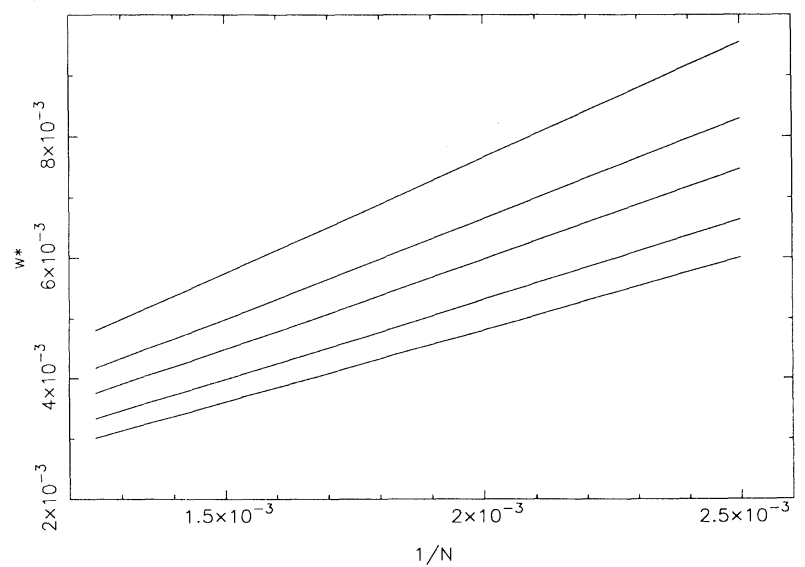

FIG. 7. Resonant frequency $\omega^{*}$ versus the inverse of the size of the lattice segment for several values of the amplitude $\epsilon$. From top to bottom the curves correspond to $\epsilon=0.05,0.2$, $0.4,0.6$, and 0.9 . For all curves it is $\phi=0$ and $p=1$. 


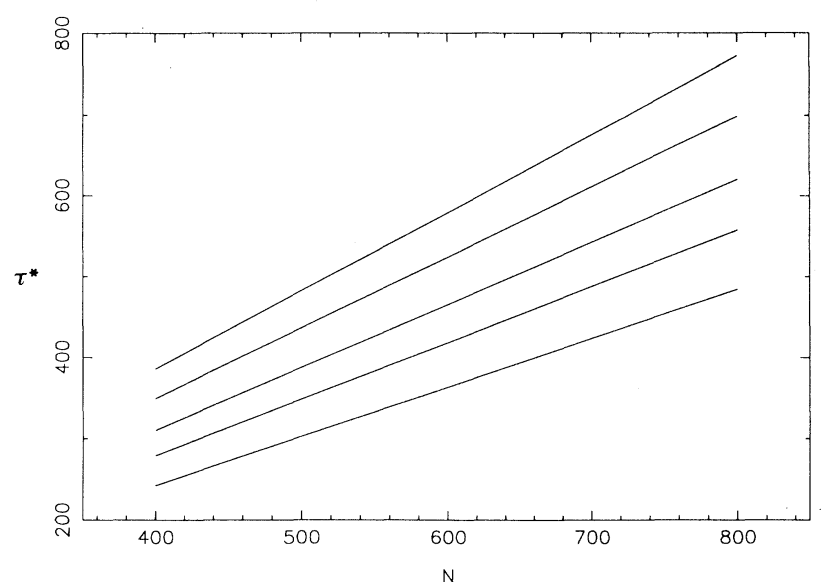

FIG. 8. (First) minimum MFPT $\tau^{*}$ plotted as a function of the size of the lattice segment for several values of the amplitude $\epsilon$. From top to bottom the curves correspond to $\epsilon=0.9,0.6,0.4,0.2$, and 0.05 . For all curves it is $\phi=0$ and $p=1$.

monotonic increasing function of the bias parameter $\epsilon$, while $\tau^{*}$ decreases with the same parameter.

Now let us examine the $\epsilon$ dependence of the MFPT. From Eq. (13) we have

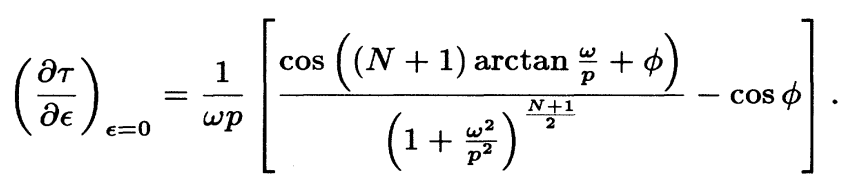

Therefore the initial slope of $\tau(\epsilon)$ will depend, among other factors, on the initial phase $\phi$ of the stimulus. Consider first the specific value $\phi=\pi / 2$, so that

$$
\left(\frac{\partial \tau}{\partial \epsilon}\right)_{\epsilon=0}=-\frac{1}{\omega p} \frac{\sin \left((N+1) \arctan \frac{\omega}{p}\right)}{\left(1+\frac{\omega^{2}}{p^{2}}\right)^{\frac{N+1}{2}}} .
$$

For fixed $N$ and $p$, the right hand side of this equation will be positive or negative depending on the argument of the sine function, that is to say, on the value of the frequency. For $\omega$ in the intervals

$$
p \tan \frac{(2 k+1) \pi}{N+1}<\omega<p \tan \frac{2(k+1) \pi}{N+1},
$$

$k=0,1,2, \ldots, k_{1}$, with $k_{1}$ the largest integer number equal to or smaller than $(N-3) / 4$, i.e., $k_{1}=[(N-3) / 4]$, the initial slope of $\tau(\epsilon)$ is positive, i.e., $\tau$ is an increasing function of $\epsilon$ for small $\epsilon$. On the other hand, for

$$
p \tan \frac{2 k \pi}{N+1}<\omega<p \tan \frac{(2 k+1) \pi}{N+1}
$$

$k=0,1,2, \ldots, k_{2}$, with $k_{2}=[(N-1) / 4], \tau(\epsilon)$ is a decreasing function for small $\epsilon$.
The transition of the MFPT, from being a decreasing function of the amplitude of a periodic cosine signal to increase with this parameter as the frequency of the signal varies, has been noticed by Gitterman and Weiss [7] for a random walk between two trapping points, i.e., the same system as in Ref. [3], but with a difference of $\pi / 2$ in the initial phase of the signal. In our model, the lowest frequency at which the transition takes place is $\omega=p \tan \frac{\pi}{N+1}$, but transitions from one regime to the other occur at all frequencies $\omega=p \tan \frac{k \pi}{N+1}$, with $k$ a positive integer. The behavior of $\tau(\epsilon)$ is quite different for $\phi=0$. The right hand side of Eq. (45) is in this case negative for all values of the frequency $\omega$ and no transition appears. This shows the important role that the initial phase of the external periodic stimulus also plays in determining the dependence of the MFPT on the amplitude of the perturbation.

Since we have discussed only the initial slope of the function $\tau(\epsilon)$, no information can be obtained about whether it is a monotonic function. We have analyzed numerically Eq. (6), and it turns out that, for those values of $\phi$ for which transitions appear, there is a frequency range near the transition frequency where $\tau$ shows a nonmonotonic behavior as $\epsilon$ is increased. An example of the described behavior is given in Fig. 9.

All the results presented in this work have been restricted to the specific initial condition given in Eq. (8), but they remain qualitatively the same for other choices. In particular, if the uniform initial condition

$$
p_{n}(0)=\frac{1}{N+1} \sum_{i=0}^{N} \delta_{n, i}
$$

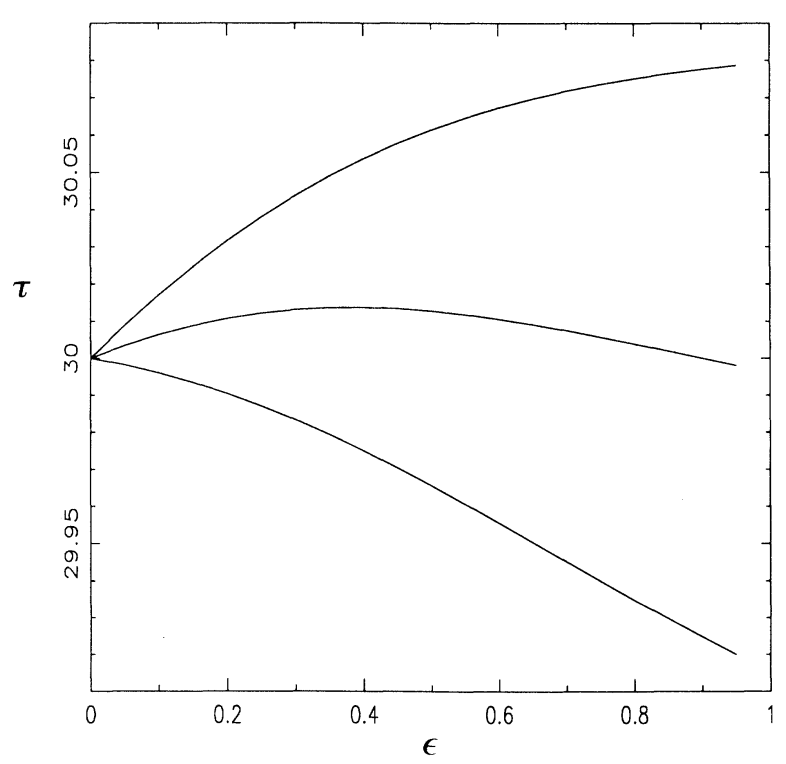

FIG. 9. MFPT versus the amplitude $\epsilon$ for $\omega=0.210$, 0.2115 , and 0.213 (top to bottom) around the second tranaction. For the three curves it is $N=29, p=1$, and $\phi=\pi / 2$. 
is considered, the main change in the behavior of the MFPT is that all the curves shown become smoother and, for instance, the peaks in Fig. 5 for $N=50$ disappear. Nevertheless, the asymptotic dependencies given by Eqs. (39) and (42) and the existence of transition frequencies as discussed above remain valid.

\section{DISCUSSION AND CONCLUDING REMARKS}

The objective in this paper has been to study the statistical properties of the residence time on a line segment for a Poisson random walk with a periodic timedependent transition rate. The simplicity of the model allows us to solve the master equation governing its evolution and to obtain an exact explicit expression for the probability distribution of the residence times. The structure of the latter shows many of the features which are considered as characteristic of resonant behavior and, in particular, of the phenomenon of stochastic resonance in bistable systems. Nevertheless, the deep physical differences between the model considered here and bistable models prevent us from trying to establish any fundamental relationship between both phenomena. First, in our model the boundary is always reached, in contrast with bistable models of stochastic resonance, where the amplitude of the perturbation is too small to produce by itself crossing of the potential barrier. In fact, the amplitude of the modulation does not play an important role in determining the resonant behavior of our system. Secondly, in Poisson processes noise and drift are controlled by the same parameter and, therefore, no competition between them is possible. This also makes a relevant difference with the random walk model studied by Bulsara, Lowen, and Rees [4].

The mean residence time exhibits a resonant behavior too, in the sense that some coherence is induced in the motion of the system, tending to reduce the time it takes the walker to reach the boundary. When the mean time is considered as a function of the frequency of the stimulus it can go through a minimum. However, this phenomenon is strongly influenced by the initial value of the perturbation. In particular, when the perturbation is maximum at the initial time it is evident that the minimum mean residence time will appear in the limit of zero frequency. Another interesting property which has been analyzed is the existence of a transition in the behavior of the mean residence time regarded as a function of the amplitude of the stimulus, passing from being an increasing function to a decreasing function of the same parameter.

In this paper we have focused on the statistics of single escape events from the line segment, without introducing any reinjection mechanism of the walker into it. Such a mechanism is necessary if we want to model firing events taking place when the walker reaches the end of the segment, as might happen, for instance, in sensory neurons [12]. The simplest possibility is to consider a deterministic instantaneous reset of the walker to its starting po- sition, resetting also the periodic stimulus to its initial value $[4,14]$. In this case, the crossing times are independent of one another and have the same distribution. As a consequence, the global random walk becomes a renewal process. An important property of renewal processes is that the power spectral density (PSD) of the response can be easily obtained from the FPTDF through the relation [15]

$$
S(\Omega)=\frac{1}{\tau^{2}} \delta\left(\frac{\Omega}{2 \pi}\right)+\frac{1}{\tau} \operatorname{Re}\left[\frac{1+\chi(\Omega)}{1-\chi(\Omega)}\right],
$$

where

$$
\chi(\Omega)=\int_{0}^{\infty} d t e^{i \Omega t} f(t) .
$$

A detailed analysis of Eq. (50) for our model will be presented in a future paper. Here we only comment on some of the relevant conclusions which come from it. The PSD corresponding to the frequency of the applied periodic stimulus $\omega$, i.e., the function $S(\Omega=\omega)$ presents a series of peaks which are approximately located at the frequencies

$$
\omega_{k}=\frac{2 k \pi p}{N}
$$

with $k$ a positive integer. The height of the peaks is a quite fast monotonic decreasing function of $k$, so that the PSD presents a clear global maximum at $\omega=\omega_{1}$. In practice, for values of $N$ not too large only a few peaks can be resolved on the scale of the highest one, i.e., of the first.

Now we consider the effect of changing $p$ while keeping constant the value of the modulation frequency. As shown in Fig. 10 for three different values of $\omega$, the PSD has a resonant behavior. There is again a series of maxima located near

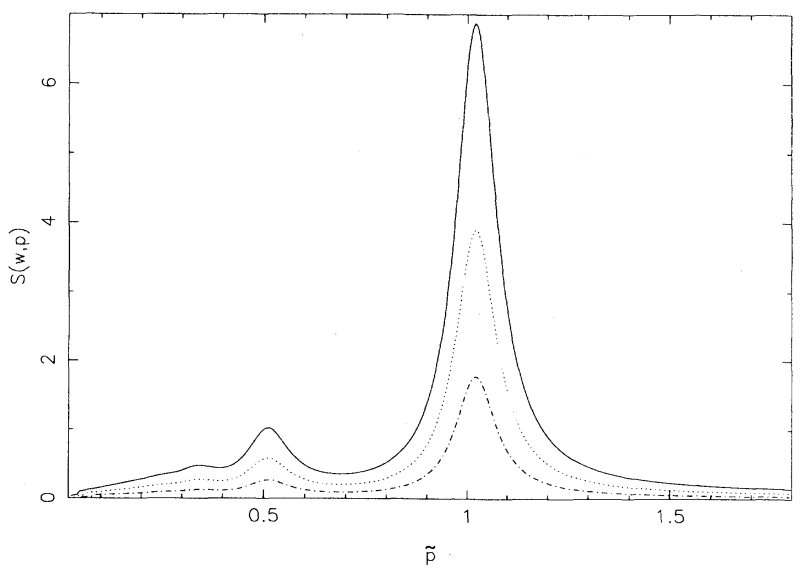

FIG. 10. Power spectral density $S(\omega)$ versus the dimensionless variable $\tilde{p}=\frac{2 \pi p}{N \omega}$ for $\omega=1$ (solid curve), $\omega=0.75$ (point curve), and $\omega=0.5$ (dashed curve). For all curves it is $N=50, \phi=\pi / 2$, and $\epsilon=0.2$. 


$$
p_{k}=\frac{\omega N}{2 k \pi},
$$

although their amplitudes decrease rapidly with increasing $k$, and a well defined absolute maximum appears for $p=\omega N / 2 \pi$. Besides, the height of a given maximum, i.e., for fixed $k$, is a monotonic increasing function of $\omega$. The above behavior of the output signal strength is qualitatively similar to what is observed in stochastic resonance in bistable systems, although, as we have already pointed out, the physical mechanisms responsible for both phenomena are different.

\section{ACKNOWLEDGMENT}

Partial support from the Dirección General de Investigación Científica y Técnica (Spain) through Grant No. PB92-0683 is gratefully acknowledged.

\section{APPENDIX A: ASYMPTOTIC ANALYSIS OF THE FPTDF}

In this appendix we are going to study the asymptotic behavior of the FPTDF given by Eq. (9) in the limit of large $N$. We introduce the dimensionless variable

$$
s=\omega t
$$

and the dimensionless function

$$
\Phi(s)=s-\frac{\epsilon}{p}[\cos (s+\phi)-\cos \phi] .
$$

From Eq. (9) we can write the distribution function for the $s$ variable as

$$
g(s)=\frac{1}{N !}\left(\frac{p}{\omega}\right)^{N+1} \Phi^{\prime}(s)[\Phi(s)]^{N} e^{-\frac{p}{\omega} \Phi(s)} .
$$

For $\epsilon$ in the interval $[0, p), \Phi(s)$ is a monotonic increasing function of $s$, while $\Phi^{\prime}(s)$ is periodic with period $2 \pi$. The function

$$
h(s)=[\Phi(s)]^{N} e^{-\frac{p}{\omega} \Phi(s)}
$$

has a maximum whose location $s_{m}$ is given by the equation

$$
\Phi\left(s_{m}\right)=\frac{\omega N}{p}
$$

Define

$$
\Psi(s)=\frac{\Phi(s)}{\Phi\left(s_{m}\right)}=\frac{p}{\omega N} \Phi(s)
$$

so that the maximum value of $h(s)$ corresponds to $\Psi(s)=$ 1 , and rewrite $g(s)$ in the form

$$
g(s)=\frac{p}{N ! \omega} N^{N} \Phi^{\prime}(s) e^{-N[\Psi(s)-\ln \Psi(s)]} .
$$

Up to this point everything is exact and no approximation has been introduced. Assume now that $N$ is very large. Expansion of $\Psi(s)-\ln \Psi(s)$ around $\Psi(s)=1$, keeping only the lowest order, yields

$$
g(s)=\frac{N^{1 / 2}}{\sqrt{2 \pi}} \Psi^{\prime}(s) e^{-\frac{N}{2}[\Psi(s)-1]^{2}},
$$

where the Stirling approximation for $N$ ! has been used. This expression is a valid approximation to Eq. (A7) for all values of $s$ for which $g(s)$ is not exponentially small. It is trivially checked that $g(s)$ as given by Eq. (A8) is normalized to 1 . To see whether it is possible to simplify further $g(s)$, consider the expansion

$$
\begin{aligned}
{[\Psi(s)-1]^{2}=} & \Psi^{\prime}\left(s_{m}\right)^{2}\left(s-s_{m}\right)^{2} \\
& +\Psi^{\prime}\left(s_{m}\right) \Psi^{\prime \prime}\left(s_{m}\right)\left(s-s_{m}\right)^{3} \\
& +\left(\frac{1}{4} \Psi^{\prime \prime}\left(s_{m}\right)^{2}+\frac{1}{3} \Psi^{\prime}\left(s_{m}\right) \Psi^{\prime \prime \prime}\left(s_{m}\right)\right) \\
& \times\left(s-s_{m}\right)^{4}+\cdots
\end{aligned}
$$

When this is introduced into Eq. (A8) and the terms of the resulting series are analyzed, it is seen that if

$$
\frac{\omega N^{1 / 2}}{p} \ll 1
$$

Eq. (A8) can be approximated by

$$
g(s)=\frac{N^{1 / 2}}{\sqrt{2 \pi} \sigma_{s}} e^{-\frac{\left(s-s_{m}\right)^{2}}{2 \sigma_{s}^{2}}},
$$

where

$$
\sigma_{s}^{2}=\frac{\omega^{2} N}{\left[p+\epsilon \sin \left(s_{m}+\phi\right)\right]^{2}}
$$

and $s_{m}$ is the solution of Eq. (A5). Upon writing Eq. (A11) we have used the fact that, in the limit defined by Eq. (A10), it is $\sigma_{s}^{2} \sim O\left(\frac{\omega^{2} N}{p^{2}}\right) \ll 2 \pi$ and, therefore, we can substitute $\Psi^{\prime}(s)$ by $\Psi^{\prime}\left(s_{m}\right)$ in Eq. (A8). In this discussion we have assumed that $\epsilon$ is not very close to $p$. Finally, one more simplification can be carried out if $\frac{\omega N}{p} \ll 1$. Then, Eq. (A5) implies that $s_{m} \ll 1$ and we have

$$
\cos \left(s_{m}+\phi\right)-\cos \phi \simeq-s_{m} \sin \phi
$$

and Eq. (A5) leads to

$$
s_{m}=\frac{\omega N}{p+\epsilon \sin \phi}
$$

In this same limit, Eq. (A12) reduces to

$$
\sigma_{s}^{2}=\frac{\omega^{2} N}{(p+\epsilon \sin \phi)^{2}}
$$

Returning to the original variable $t$, the expressions in Eqs. (14)-(20) are obtained. 


\section{APPENDIX B: LOCATION OF THE PEAKS OF THE FPTDF}

We start from Eq. (A7), i.e.,

$$
g(s)=\frac{p}{N ! \omega} N^{N} \Phi^{\prime}(s) \Xi(s),
$$

where

$$
\Xi(s)=\exp \{-N[\Psi(s)-\ln \Psi(s)]\} .
$$

Differentiation of Eq. (B1) yields

$$
\frac{\partial \ln g(s)}{\partial s}=\frac{\Phi^{\prime \prime}(s)}{\Phi^{\prime}(s)}+\frac{\partial \ln \Xi(s)}{\partial s} .
$$

Since $\Phi^{\prime}(s)>0$ for all $s$, the stationary points of $g(s)$ will be given by the roots of the equation

$$
\Phi^{\prime \prime}(s)+\Phi^{\prime}(s) \frac{\partial \ln \Xi(s)}{\partial s}=0,
$$

or, using Eq. (A2),

$$
\cos (s+\phi)+\frac{p}{\epsilon}\left(1+\frac{\epsilon}{p} \sin (s+\phi)\right) \frac{\partial \ln \Xi(s)}{\partial s}=0 .
$$

Let us now assume we are in a region of $s$ where

$$
\left|\frac{\partial \ln \Xi(s)}{\partial s}\right| \leq \gamma,
$$

where $\gamma$ is some positive parameter, and define

$$
\frac{\partial \ln \Xi(s)}{\partial s}=\gamma \zeta(s),
$$

with $|\zeta(s)| \leq 1$ for the values of $s$ under consideration. In terms of this new function Eq. (B5) reads

$$
\cos (s+\phi)+\frac{p \gamma}{\epsilon}\left(1+\frac{\epsilon}{p} \sin (s+\phi)\right) \zeta(s)=0 .
$$

Consider the equation

$$
\cos (s+\phi)=0,
$$

whose solutions are (we restrict ourselves to the maxima of the sine function)

$$
s_{l}^{(0)}=2 \pi l+\frac{\pi}{2}-\phi
$$

$l=1,2, \ldots$. If we write $s_{l}=s_{l}^{(0)}+x s_{l}^{(1)}+\cdots$, where $x=p \gamma / \epsilon$, it is obtained from Eq. (B8) that

$$
s_{l}^{(1)}=\left(1+\frac{\epsilon}{p}\right) \zeta\left(s_{l}^{(0)}\right)
$$

and it is $\left|s_{l}^{(1)}\right|<2$. The condition which $s_{l}^{(0)}$ must verify in order to be a good approximation to $s_{l}$ is $x \ll 1$, or

$$
\frac{p \gamma}{\epsilon} \ll 1
$$

It is easily seen that this also implies that the peaks are well defined, in the sense that

$$
\left|s_{l}-s_{l}^{(0)}\right| \ll\left|s_{l+1}^{(0)}-s_{l}^{(0)}\right| .
$$

Let us try to make this condition more transparent. Using the definition of $\Xi(s)$, Eq. (B2), we have

$$
\frac{\partial \ln \Xi(s)}{\partial s}=-\frac{\Phi^{\prime}(s) p}{\Phi(s) \omega}\left(\Phi(s)-\frac{\omega N}{p}\right) .
$$

As $\Phi\left(s_{m}\right)=\omega N / p$ and $\Phi(s)$ is a monotonic increasing function of $s$, it follows that $\left|\Phi(s)-\frac{\omega N}{p}\right|$ monotonically increases when one moves away from $s_{m}$ in either of the two directions. For $s$ inside the interval $\left[s_{m}-\Delta, s_{m}+\Delta\right]$, it is

$$
\left|\frac{\partial \ln \Xi(s)}{\partial s}\right| \leq \frac{\left(1+\frac{\epsilon}{p}\right)^{2} \frac{\Delta p^{2}}{\omega^{2} N}}{1-\left(1+\frac{\epsilon}{p}\right) \frac{p \Delta}{\omega N}}
$$

if $\left(1+\frac{\epsilon}{p}\right) \frac{p \Delta}{\omega N}<1$. By using this result, Eq. (B12) becomes

$$
\frac{p^{3}}{\omega^{2} N \epsilon}\left|s_{l}-s_{m}\right| \ll 1,
$$

which is equivalent to Eq. (22).
[1] B. McNamara and K. Wiesenfeld, Phys. Rev. A 39, 4854 (1989).

[2] P. Jung, Phys. Rep. 234, 175 (1994).

[3] J. E. Fletcher, S. Havlin, and G. H. Weiss, J. Stat. Phys. 51, 215 (1988).

[4] A. R. Bulsara, S. B. Lowen, and C. D. Rees, Phys. Rev. E 49, 4989 (1994).

[5] J. L. Viovy, Electrophoresis 10, 429 (1990).

[6] A. Bulsara and G. Schmera, Phys. Rev. E 47, 3734
(1993), and references therein.

[7] M. Gitterman and G. H. Weiss, J. Stat. Phys. 74, 941 (1994).

[8] N. G. van Kampen, Stochastic Processes in Physics and Chemistry, 2nd ed. (North-Holland, Amsterdam, 1992).

[9] C. W. Gardiner, A Handbook of Stochastic Methods, 2nd ed. (Springer-Verlag, New York, 1985).

[10] J. J. Brey and J. Casado-Pascual, Physica A 212, 123 (1994). 
[11] C. R. Doering and J. C. Gadoua, Phys. Rev. Lett. 69, 2318 (1992).

[12] H. Tuckwell, Stochastic Processes in the Neurosciences (SIAM, Philadelphia, 1979).

[13] Handbook of Mathematical Functions, edited by $M$.
Abramowitz and I. Stegun (Dover, New York, 1972).

[14] K. Wiesenfeld, D. Pierson, E. Pantazelou, C. Dames, and F. Moss, Phys. Rev. Lett. 72, 2125 (1994).

[15] T. Lukes, Proc. Phys. Soc. London 78, 153 (1961). 